\title{
A GENERALISATION OF A FORMULA DUE TO SCHUBERT
}

\author{
by J. G. BRENNAN \\ (Received 6th May 1957)
}

1. Let there be given, on an algebraic curve $C$, of genus $p$, a linear series $g_{m}^{r}$ and an algebraic series $\gamma_{n}^{1}$ of index $\nu$, both without fixed points. The number of groups of $r+1$ points which are common to a set of $g_{m}^{r}$ and a set of $\gamma_{n}^{1}$ has been shown by Schubert (1) to be

$$
m \nu\left(\begin{array}{c}
n-1 \\
r
\end{array}\right)-\frac{1}{2}\left(\begin{array}{c}
n-2 \\
r-1
\end{array}\right) d
$$

where $d$ is the number of double points of $\gamma_{n}^{1}$.

The object of this note is to generalise the above result by seeking the number of groups of $s=\sum_{i=1}^{p} \alpha_{i}$ points which are common to a set of $g_{m}^{r}$ and a set of $\gamma_{n}^{1}$, these $s$ points consisting of a batch of $\alpha_{1}$ points of multiplicity $k_{1}$, a batch of $\alpha_{2}$ points of multiplicity $k_{2}, \ldots$, and a batch of $a_{\rho}$ points of multiplicity $k_{\rho}$ for the set of $g_{m}^{r}$ which contains them, and being all simple points of the set of $\gamma_{n}^{1}$ which contains them. In order that there shall be a finite number of such groups of points it is necessary that $r+1=\sum_{i=1}^{\rho} \alpha_{i} k_{i}$. The number sought may then be denoted by

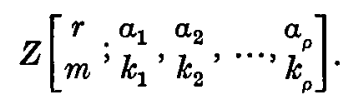

2. Consider an algebraic series $\gamma_{n}^{1}$ and a linear series $g_{m+l-k_{1}+1}^{r+l-k_{1}+1}$ both on $C$, where $l<k_{1}<k_{2}<\ldots<k_{\rho}$.

A general point $P$ of $C$ belongs to $\nu$ sets of $\gamma_{n}^{1}$. If $Q$ be any one of the points of these sets, other than $P$, there is defined a correspondence $(P, Q)$, with indices $v(n-1)$, whose united points are the $d$ double points of $\gamma_{n}^{1}$.

From the $n-1$ points $Q$ of such a set, a batch of $\left(a_{1}-1\right)$ points $Q_{1}$, a batch of $a_{2}$ points $Q_{2}, \ldots$, and a batch of $a_{\rho}$ points $Q_{\rho}$ may be chosen in

$$
\left(\begin{array}{c}
n-1 \\
a_{1}-1, a_{2}, \ldots, a_{\rho}
\end{array}\right)=\frac{(n-1) !}{\left(a_{1}-1\right) ! a_{2} ! \ldots a_{\rho} !(n-s) !}
$$

ways. Each such choice defines a set of $g_{m+l-k_{1}+1}^{r+l-k_{1}+1}$ for which $P$ is an $l$-ple point and which has each point $Q_{i}$ as a $k_{i}$-ple point $(i=1,2, \ldots, \rho)$. Let this be done for each of the $v$ sets of $\gamma_{n}^{1}$ defined by $P$. Then if $R$ be any one of the further points of $g_{m+l-k_{1}+1}^{++l-k_{1}+1}$ thus defined, there is established a correspondence $(P, R)$ whose second index is

$$
v\left(\begin{array}{c}
n-1 \\
a_{1}-1, a_{2}, \ldots, a_{p}
\end{array}\right)(m-r)
$$


and whose first index is $N_{l}$ where

$$
N_{l}=Z\left[\begin{array}{ccc}
r+l-k_{1} & 1 \\
m+l-k_{1} & l, & a_{1}-1 \\
k_{1}
\end{array}, \frac{a_{2}}{k_{2}}, \ldots, \frac{a_{\rho}}{k_{\rho}}\right] \text { if } l \neq 0,
$$

while

$$
N_{0}=(n-s+1) Z\left[\begin{array}{ccc}
r-k_{1} \\
m-k_{1}
\end{array} ; \begin{array}{ccc}
a_{1}-1 & a_{2} & k_{1} \\
k_{2}
\end{array}, \ldots, \frac{a_{\rho}}{k_{\rho}}\right] \text {. }
$$

The united points of $(P, R)$ are $U_{l}$ in number where

$$
U_{l}=Z\left[\begin{array}{cccc}
r+l-k_{1}+1 & 1 \\
m+l-k_{1}+1 & ;+1
\end{array}, \begin{array}{ccc}
a_{1}-1 & a_{2} \\
k_{1} & , & k_{2}
\end{array}, \ldots, \frac{a_{\rho}}{k_{p}}\right] \text { if } l \neq k_{1}-1
$$

while

$$
U_{k_{1}-1}=a_{1} Z\left[\begin{array}{c}
r \\
m
\end{array} ; \begin{array}{l}
a_{1} \\
k_{1}
\end{array}, \ldots, \frac{a_{p}}{k_{\rho}}\right]
$$

3. The sets of $g_{m+l-k_{1}+1}^{r+l-k_{1}+1}$ defined above contain, in addition to the points $R$, the point $P$ counted $\nu l\left(\begin{array}{c}n-1 \\ a_{1}-1, a_{2}, \ldots, a_{\rho}\end{array}\right)$ times and the points $Q$ each counted

$$
\begin{gathered}
k_{1}\left(\begin{array}{c}
n-2 \\
a_{1}-2, a_{2}, \ldots, a_{\rho}
\end{array}\right)+\sum_{i=2}^{\rho} k_{i}\left(\begin{array}{c}
n-2 \\
a_{1}-1, a_{2}, \ldots, a_{i-1}, a_{i}-1, a_{i+1}, \ldots, a_{\rho}
\end{array}\right) \\
=\frac{r-k_{1}+1}{n-1}\left(\begin{array}{c}
n-1 \\
a_{1}-1, a_{2}, \ldots, a_{\rho}
\end{array}\right)
\end{gathered}
$$

times. It follows that the correspondence

$$
T_{l} \equiv \frac{r-k_{1}+1}{n-1}\left(\begin{array}{c}
n-1 \\
a_{1}-1, a_{2}, \ldots, a_{\rho}
\end{array}\right)(P, Q)+(P, R)
$$

has valency

$$
\nu l\left(\begin{array}{c}
n-1 \\
a_{1}-1, a_{2}, \ldots, a_{\rho}
\end{array}\right)
$$

Its indices are

$$
\left(\begin{array}{c}
n-1 \\
a_{1}-1, a_{2}, \ldots, a_{\rho}
\end{array}\right)\left(r-k_{1}+1\right) \nu+N_{l} \text { and }\left(\begin{array}{c}
n-1 \\
a_{1}-1, a_{2}, \ldots, a_{\rho}
\end{array}\right)\left(m-k_{1}+1\right) \nu
$$

whose sum is

$$
v\left(\begin{array}{c}
n-1 \\
a_{1}-1, a_{2}, \ldots, a_{\rho}
\end{array}\right)\left[m+r-2\left(k_{1}-1\right)\right]+N_{l}
$$

and so, by the Cayley-Brill theorem (2)

$$
U_{l}-N_{l}=\left(\begin{array}{c}
n-1 \\
a_{1}-1, a_{2}, \ldots, a_{\rho}
\end{array}\right)\left\{\nu\left[m+r+2\left(l p-k_{1}+1\right)\right]-\frac{r-k_{1}+1}{n-1} d\right\} .
$$


On giving $l$ the values $k_{1}-1, k_{1}-2, \ldots, 1,0$ in succession, and adding the resulting $k_{1}$ relations, the $k_{1}-1$ numbers

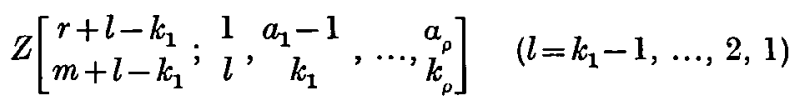

disappear, leaving

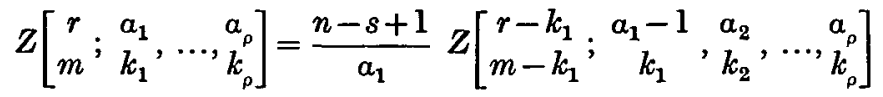

$$
\begin{aligned}
& +\left(\begin{array}{c}
n \\
a_{1}, \ldots, a_{\rho}
\end{array}\right)\left\{\frac{\nu k_{1}}{n}\left[m+r+(p-2)\left(k_{1}-1\right)\right]-\frac{\left(r-k_{1}+1\right) k_{1} d}{n(n-1)}\right\} \text {. }
\end{aligned}
$$

4. A $g_{m}^{k-1}$ on $C$ contains $k[m+(p-1)(k-1)]$ sets each possessing a point of multiplicity $k(3)$, and since this point belongs to $\nu$ sets of $\gamma_{n}^{1}$ it follows that

$$
Z\left[\begin{array}{cc}
k-1 & 1 \\
m
\end{array}, k\right]=v k[m+(p-1)(k-1)] .
$$

The above recurrence relation now permits the successive calculation of

$$
Z\left[\begin{array}{cc}
a_{\rho} k_{\rho}-1 & ; \\
m & a_{\rho} \\
k_{\rho}
\end{array}\right], Z\left[\begin{array}{ccc}
a_{\rho-1} k_{\rho-1}+a_{\rho} k_{\rho}-1 & ; \\
m & a_{\rho-1}, a_{\rho} \\
k_{\rho-1} & k_{\rho}
\end{array}\right]
$$

and so on. Thus it is found that

$$
Z\left[\begin{array}{cc}
a k-1 & a \\
m & k
\end{array}\right]=v k\left(\begin{array}{c}
n-1 \\
a-1
\end{array}\right)\{m+(k-1)(p-1)\}-\frac{1}{2}\left(\begin{array}{c}
n-2 \\
a-2
\end{array}\right) k^{2} d,
$$

which clearly reduces to Schubert's formula when $k=1$. It may now be verified by induction that

$$
\begin{aligned}
& Z\left[\begin{array}{r}
r \\
m
\end{array} ; \begin{array}{l}
a_{1} \\
k_{1}
\end{array}, \ldots, \begin{array}{l}
a_{\rho} \\
k_{\rho}
\end{array}\right]=\left(\begin{array}{c}
n \\
a_{1}, \ldots, a_{\rho}
\end{array}\right)\left[\frac{v}{n}\left\{(r+1)(m-p+1)+(p-1) \sum_{i=1}^{\rho} a_{i} k_{i}{ }^{2}\right\}\right. \\
&\left.-\frac{d}{2 n(n-1)}\left\{(r+1)^{2}-\sum_{i=1}^{\rho} a_{i} k_{i}{ }^{2}\right\}\right] .
\end{aligned}
$$

5. By way of illustration, consider the following problem. Let $C$ be a plane curve, of order $n \geqslant 3$, and genus $p$. There exists a single infinity of conics each of which osculates $C$ at two points. What is the class of the envelope of the line of join of these points ?

The totality of conics cut a $g_{2 n}^{5}$ on $C$, while the lines through a general point of the plane cut a $g_{n}^{1}$. Hence, setting

$$
m=2 n, r=5, \nu=1, d=2(n+p-1), \rho=1, a_{1}=2, k_{1}=3,
$$

the number sought is

$$
Z\left[\begin{array}{cc}
5 & 2 \\
2 n & 3
\end{array}\right]=3(2 n-5)(n+p-1)
$$


For example, if $n=3$ and $p=1$ the envelope is of class 9 . It is easy to show that the envelope in this case degenerates into the nine inflexions of the cubic.

The writer wishes to thank the referee for a number of helpful comments.

\section{REFERENCES}

(1) H. F. Baker, Principles of Geometry, vol. VI, Cambridge University Press, 1925, p. 37.

(2) H. F. Baker, ibid., pp. 8, 9.

(3) H. F. Baker, ibid., p. 10.

Department of Mathematics

University CoLlege of Swansea 